\title{
Probing sterile neutrinos in the framework of inverse seesaw mechanism through leptoquark productions
}

\author{
Debottam Das, Kirtiman Ghosh, ${ }^{\dagger}$ and Manimala Mitra ${ }^{\ddagger}$ \\ Institute of Physics, Bhubaneswar 751005, India \& Homi Bhabha National Institute, \\ Training School Complex, Anushakti Nagar, Mumbai 400085, India \\ Subhadeep Mondal ${ }^{\S}$ \\ Regional Centre for Accelerator-based Particle Physics, Harish-Chandra Research Institute, HBNI, \\ Chhatnag Road, Jhunsi, Allahabad 211019, India and Department of Physics, \\ and Helsinki Institute of Physics, P. O. Box 64, FI-00014 Helsinki, University of Helsinki, Finland
}

(Received 1 September 2017; published 31 January 2018)

\begin{abstract}
We consider an extension of the standard model (SM) augmented by two neutral singlet fermions per generation and a leptoquark. In order to generate the light neutrino masses and mixing, we incorporate inverse seesaw mechanism. The right-handed neutrino production in this model is significantly larger than the conventional inverse seesaw scenario. We analyze the different collider signatures of this model and find that the final states associated with three or more leptons, multijet and at least one $b$-tagged and (or) $\tau$-tagged jet can probe larger RH neutrino mass scale. We have also proposed a same-sign dilepton signal region associated with multiple jets and missing energy that can be used to distinguish the present scenario from the usual inverse seesaw extended SM.
\end{abstract}

DOI: 10.1103/PhysRevD.97.015024

\section{INTRODUCTION}

ATLAS and CMS Collaborations have already made huge impacts to unravel any possible signatures of physics beyond the standard model (BSM) at the LHC [1,2]. Although no signature of new particles has yet been found by the direct search, there are many reasons for pursuing our search in BSM physics at the LHC. Undoubtedly, one of the most compelling motivations comes from the existence of nonzero neutrino masses and substantial mixing among the three light neutrino states (see for example, $[3,4])$. In this frontier, the neutrino oscillation experiments have made significant progress to measure the mass square difference and the mixing angles of the neutrinos with unprecedented precision, which indicates that at least two of the light neutrino states have to have tiny nonzero masses. As a natural consequence, many proposals have been put forward over the years to accommodate the neutrino masses and mixing in the theory of which

\footnotetext{
*debottam@iopb.res.in

†kirti.gh@gmail.com

*manimala@iopb.res.in

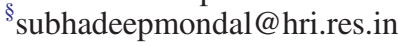

Published by the American Physical Society under the terms of the Creative Commons Attribution 4.0 International license. Further distribution of this work must maintain attribution to the author(s) and the published article's title, journal citation, and DOI. Funded by SCOAP ${ }^{3}$. various seesaw mechanisms have remained of primary interest [5-16].

The simplest seesaw extension, known as the type-I seesaw mechanism [5-8] is accompanied with additional Majorana right-handed neutrinos $\left(\nu_{R i}\right)$. Light neutrinos gain tiny nonzero masses by virtue of their mixings with these heavy neutrino states. In such scenarios, the mass scale of these Majorana neutrinos has to lie very close to gauge coupling unification scale $M_{G} \sim 10^{16} \mathrm{GeV}$ in order to account for the tiny neutrino masses. Such a massive $\nu_{R}$ completely decouples from the low energy theory and remains out of the kinematic reach of the LHC. One can, of course, bring down this mass scale to $\mathrm{TeV}$ range, but at the cost of a very small Dirac neutrino Yukawa coupling $\left(\sim 10^{-6}\right)$ which again makes any possible phenomenological aspects of the heavy neutrinos unforeseeable at the LHC. ${ }^{1}$ Thus the lack of predictability of this simplistic scenario has forced theorists as well as experimentalists to study models which can readily be probed at the LHC with the existing data. One such scenario is called the inverse seesaw mechanism [18-20] where the SM particle content is augmented by two singlet neutrinos with opposite lepton numbers $(+1$ and -1$)$. The generic form of the light neutrino mass can be expressed as $m_{\nu} \sim\left(m_{D}^{2} / M_{R}^{2}\right) \mu$, where

\footnotetext{
${ }^{1}$ Such small couplings can only be probed with very high precision colliders and naturally lepton colliders are better suited to serve the purpose [17].
} 
$m_{D} \sim Y_{\nu} v$ represents the Dirac neutrino mass term, $v$, the electroweak VEV, $Y_{\nu}$, a generic Yukawa coupling and $\mu$, a lepton number violating $(\Delta L=2)$ mass parameter which can be kept naturally small [21]. The presence of this small ( $\sim \mathrm{eV}) \Delta L=2$ mass term helps to keep the sterile neutrino mass scale $M_{R}$ close to TeV, i.e., within the reach of LHC, with order one Yukawa coupling. This feature of the model leads to a plethora of testable phenomenological consequences [22-32] which have been studied quite extensively in the context of present and future collider experiments [33-42]. For the RH neutrino production through vector boson fusion and higher order corrections, see [43,44]. Note that, although the heavy neutrino states now lie within the kinematic reach of the LHC, the production processes are still driven by the charged/neutral current weak interactions, through active-sterile neutrino mixing, that depend crucially on the mass scale of the RH neutrinos $M_{R}$. Hence, the cross section falls rapidly with increasing heavy neutrino masses and with smaller mixings. The production cross section of the RH neutrinos can be drastically enhanced in presence of leptoquarks (LQ). Leptoquarks, being charged under $S U(3)_{C}$, are copiously produced at the LHC. The right-handed neutrinos can result from the decays of LQs. It is important to note that in this case, the RH neutrino production does not depend on the activesterile mixing. Therefore, $\mathrm{RH}$ neutrinos can be probed at the LHC irrespective of their mixing with the active component as long as they decay inside the detector.

Introduction of LQ (for a recent review see [45]) to the SM Lagrangian is motivated from a quite different viewpoint. In fact, in the Pati-Salam model that LQs are a natural outcome of unification of quarks and leptons [46]. The presence of these new exotic particles has been further motivated by the simple grand unified gauge groups of $S U(5)$ [47] and $S O(10)[48,49]$. While both vector (spinone) and scalar LQ (spin-zero) states are possible in local quantum field theories, the scalar states are more attractive as they do not lead to any potential problems related to loop corrections [50,51]. Moreover, it has recently been shown that the scalar LQs are indeed very useful to explain various $B$-physics anomalies like $R_{K}$ [52] or $R_{D^{*}}$ [53]. Explanation of both $R_{K}$ and $R_{D}$ anomalies with a scalar LQ with the hypercharge $Y=\frac{1}{6}$ would be possible if one includes a new interaction between a scalar LQ and a right-handed neutrino [51]. Keeping in mind that the presence of a LQ- $\nu_{R}$ coupling is highly motivated to accommodate $B$-physics anomalies, in this work we will go one step further. We will introduce additional sterile neutrinos in the model to comply with all existing experimental data on light neutrino mass and mixing angles while the mass of the heavy neutrino states have been kept smaller than the leptoquark states. In practice, we incorporate inverse seesaw to the SM extended by leptoquarks for our study. We show that the heavy neutrinos can be copiously generated from the decays of the scalar LQs which can be produced via strong interactions thanks to their $S U(3)$ interactions. The relevant couplings and masses of the LQs and the heavy neutrinos are chosen in a way such that they are consistent with the experimental constraints and at the same time maximize the heavy neutrino productions from the cascade decay. Moreover our collider study shows that such a scenario has the potential of probing heavy neutrino masses up to a much higher range compared to that in the usual neutrino mass models.

The paper is organized as follows. In Sec. II, we introduce the model. Following that, in Sec. III, we discuss the present experimental constraints on the RH neutrinos and the LQs. The collider signatures have been discussed in Sec. IV. Finally, we present our conclusion.

\section{INVERSE SEESAW MECHANISM IN THE SM EXTENDED BY LEPTOQUARKS}

The Lagrangian which we will consider in this work can be cast as

$$
\mathcal{L}=\mathcal{L}_{\mathrm{SM}}+\mathcal{L}_{\Delta}+\mathcal{L}_{I S}
$$

$\mathcal{L}_{\Delta}$ includes the interaction terms between the leptoquarks and the SM particles while $\mathcal{L}_{I S}$ comprises of the relevant terms for generation of the neutrino mass and mixing angles. The association between $\Delta$ and $\mathcal{L}_{I S}$ have been realized through right-handed neutrino states $\nu_{R i}$ whose mass can vary $\mathcal{O}(1) \mathrm{eV} \leq M_{R i} \leq \mathcal{O}(1) \mathrm{TeV}$. Moreover the inverse seesaw extension of the SM requires three new fermionic singlet fields $X_{i}(i=1,2,3)$ with lepton numbers +1 in contrast to the $\nu_{R i}$ states of lepton numbers -1 , respectively. ${ }^{2}$

$$
\mathcal{L} \in \varepsilon_{a b} Y_{\nu}^{i j} \nu_{i}^{R} L_{j}^{a} H^{b}+M_{R_{i}} \nu_{R i} X_{i}+\frac{1}{2} \mu_{X_{i j}} X_{i} X_{j},
$$

where $i, j=1,2,3$ denote generation indices. In the above $L_{i}$ denotes three generation SU(2) lepton doublets. $M_{R_{i}}$ represents the right-handed neutrino bilinear term which conserves lepton number. The total lepton number $L$ is no longer a good quantum number because of nonvanishing $\mu_{X}$, though $(-1)^{L}$ is still a good symmetry. Clearly, as $\mu_{X_{i}} \rightarrow 0$, lepton number conservation is restored, since $M_{R}$ does not violate lepton number.

The Leptoquark part of the Lagrangian for a scalar LQ state $\Delta$ transforms as $\Delta \in\left(3,2, \frac{1}{6}\right)$ under the SM gauge group can be written as $[45,51]$

$$
\mathcal{L}_{\Delta}=\bar{d}_{R} Y_{L}(\tilde{\Delta})^{\dagger} L+\bar{Q} Y_{R} \Delta \nu_{R}+\text { H.c.; }
$$

where $\tilde{\Delta}=i \sigma_{2} \Delta^{*}$. Defining mass eigenstates for a fermionic field $\psi_{L, R}^{i}$ as $\psi_{L, R}^{m i}=U_{L, R}^{i} \psi_{L, R}^{i}$ [51], one can get

\footnotetext{
${ }^{2}$ Note that in the minimal inverse seesaw extension of the SM, two pairs of the singlet field would be sufficient to satisfy all neutrino data [54].
} 
(for simplicity we remove the superscript $m$ from the fields in mass basis.)

$$
\begin{aligned}
\mathcal{L}_{\Delta}= & Y_{L}^{i j} \bar{d}_{R}^{i} U_{\mathrm{PMNS}}^{j k} \nu_{L}^{k} \Delta^{(-1 / 3)}-Y_{L}^{i j} \bar{d}_{R}^{i} \ell_{L}^{j} \Delta^{(2 / 3)} \\
& +Y_{R}^{i j} \bar{u}_{L}^{i} \nu_{R}^{j} \Delta^{(2 / 3)}+Y_{R}^{i j} \bar{d}_{L}^{k} V_{\mathrm{CKM}}^{k i} \nu_{R}^{j} \Delta^{(-1 / 3)}+\text { H.c. },
\end{aligned}
$$

where $Y_{L} \rightarrow U_{R}^{d \dagger} Y_{L} U_{L}^{\ell}$ and $Y_{R} \rightarrow U_{L}^{u \dagger} Y_{R} U_{R}^{\nu}$ have been used. Similarly, $U_{\mathrm{PMNS}}=U_{L}^{\ell} U_{L}^{\nu^{\dagger}}$, and $V_{\mathrm{CKM}}=U_{L}^{d} U_{L}^{u \dagger}$ represent the Pontecorvo-Maki-Nakagawa-Sakata and the Cabibbo-Kobayashi-Maskawa matrices. Now assuming the Yukawa couplings to the first generations of the quarks/ leptons as zero to become consistent with the atomic parity violation experiments [45], we represent the matrices as follows [51]:

$$
Y_{L, R}=\left(\begin{array}{ccc}
0 & 0 & 0 \\
0 & Y_{L, R}^{22} & Y_{L, R}^{23} \\
0 & Y_{L, R}^{32} & Y_{L, R}^{33}
\end{array}\right) .
$$

Here we primarily consider productions of $\Delta^{(2 / 3)}$ states because of its direct coupling with $t$ quarks and the third generation RH neutrinos $\nu_{R_{3}}$. In the following we consider two scenarios. (i) Yukawa couplings that can potentially explain the $B$-physics anomalies. Here $Y_{L, R}^{2 i}$ can be small, while $Y_{L, R}^{3 i}$ can be large. In particular, there is a lower bound on $Y_{L}^{33} \geq 1.5$ [55] which we will use for our calculation (ii) Yukawa couplings of the second and third generations are unconstrained from $B$ physics, though the choice of $Y_{R}^{33}$ is completely driven by the fact that we need to maximize the branching ratio LQ $\rightarrow t N_{\tau}$.

\section{A. Neutrino masses in inverse seesaw}

We consider a general framework with three generations for the sterile neutrinos, namely $\nu_{R i}$ and $X_{i}$. Consequently, one has the following symmetric $(9 \times 9)$ mass matrix $\mathcal{M}$ in the basis $\left\{\nu, \nu_{R}, X\right\}$,

$$
\mathcal{M}=\left(\begin{array}{ccc}
0 & m_{D}^{T} & 0 \\
m_{D} & 0 & M_{R} \\
0 & M_{R}^{T} & \mu_{X}
\end{array}\right),
$$

Where, $m_{D}=\frac{1}{\sqrt{2}} Y_{\nu} v$ and $M_{R}, \mu_{X}$ are $(3 \times 3)$ matrices in family space. Assuming $\left(m_{D}, \mu_{X} \ll M_{R}\right)$ the diagonalization results in an effective Majorana mass matrix for the light neutrinos

$m_{\nu}=m_{D}^{T} M_{R}^{T-1} \mu_{X} M_{R}^{-1} m_{D}=\frac{v^{2}}{2} Y_{\nu}^{T}\left(M_{R}^{T}\right)^{-1} \mu_{X} M_{R}^{-1} Y_{\nu}$.

The most important aspect of the inverse seesaw mechanism is that the smallness of the light neutrino masses is directly controlled by the scale of $\mu_{X}$. Having this small dimensionful term in the Lagrangian is technically natural in the sense of 't Hooft [21], as in the limit of vanishing $\mu_{X}$ one recovers the lepton number symmetry. The lepton number conserving mass parameters $m_{D}$ and $M_{R}$ can easily accommodate large (natural) Yukawa couplings $\left(Y_{\nu} \sim \mathcal{O}(1)\right)$ and a right-handed neutrino mass scale around the TeV, see Eq. (6). In analogy to a type-I seesaw, one can define an effective right-handed neutrino mass term $M$ such that

$$
M^{-1}=\left(M_{R}^{T}\right)^{-1} \cdot \mu_{X} \cdot M_{R}^{-1} .
$$

With this definition, the light neutrino mass matrix can be cast in a way which strongly resembles a standard (type-I) seesaw equation,

$$
m_{\nu}=\frac{v^{2}}{2} Y_{\nu}^{T} M^{-1} Y_{\nu}
$$

This effective light neutrino mass matrix $\left(m_{\nu}\right)$ can be diagonalized as

$$
U_{\mathrm{PMNS}}^{T} m_{\nu} U_{\mathrm{PMNS}}=\operatorname{diag} m_{i} .
$$

In the subsequent analysis, we assume $M_{R}$ and $Y_{\nu}$ are $\operatorname{diagonal}\left(M_{R_{i j}}=\operatorname{diag} M_{R_{i i}}, Y_{\nu_{i j}}=\operatorname{diag} Y_{\nu_{i i}}\right)$. This choice of $\operatorname{diag} Y_{\nu_{i i}}$ naturally ameliorates constraints from lepton flavor changing processes. However, $\mu_{X i j}$ is not flavor diagonal and its structure can be determined by using Eqs. (7), (8), and (9) where the present results on neutrino data [4] have been used.

In our analysis, we have used the best-fit values of the mass square differences and the mixing angles to fit $\mu_{X}$. The light neutrino masses and the PMNS mixing matrix have been depicted in Table I.

\section{B. Production and decay of Leptoquarks}

Being triplet under $S U(3)_{C}$, leptoquarks have gauge couplings with gluons. Therefore, at the LHC, leptoquarks

\begin{tabular}{|c|c|c|c|}
\hline Parameters & \multicolumn{3}{|c|}{ Values } \\
\hline $\sin ^{2} \theta_{12}$ & \multicolumn{3}{|c|}{$0.304_{-0.012}^{+0.013}$} \\
\hline $\sin ^{2} \theta_{23}$ & \multicolumn{3}{|c|}{$0.452_{-0.028}^{+0.052}$} \\
\hline $\sin ^{2} \theta_{13}$ & \multicolumn{3}{|c|}{$0.0218_{-0.001}^{+0.001}$} \\
\hline$\Delta m_{21}^{2} \mathrm{eV}^{2}$ & \multicolumn{3}{|c|}{$\left(7.50_{-0.17}^{+0.19}\right) \times 10^{-5}$} \\
\hline$\Delta m_{32}^{2} \mathrm{eV}^{2}$ & \multicolumn{3}{|c|}{$\left(2.457_{-0.047}^{+0.047}\right) \times 10^{-3}$} \\
\hline \multirow{3}{*}{ PMNS [4] } & $0.801-0.845$ & $0.225-0.517$ & $0.246-0.529)$ \\
\hline & $0.514-0.580$ & $0.441-0.699$ & $0.464-0.713$ \\
\hline & $0.137-0.158$ & $0.614-0.793$ & $0.590-0.776)$ \\
\hline
\end{tabular}

TABLE I. Three-flavor neutrino oscillation data obtained from global fit for normal hierarchy in neutrino masses and the PMNS matrix elements with their $3 \sigma$ allowed ranges. We have only used the best-fit values of the mass square differences and the mixing angles to fit an off-diagonal $\mu_{X}$ in order to fit the neutrino oscillation data. 
are produced in pairs via gluon-gluon and quark-antiquark initiated processes. Gluon-gluon initiated processes primarily proceed through a leptoquark exchange in the $t(u)$-channel or a gluon exchange in the $s$-channel. On the other hand, quark-antiquark initiated processes take place only via a gluon exchange in the $s$-channel and hence, suppressed compared to the $t(u)$-channel leptoquark exchange. Leptoquark can also couple to a quark-lepton pair via Yukawa interactions as shown in Eq. (4). Therefore, quark-antiquark initiated processes may also proceed through a lepton (charged or neutral) exchange in the $t(u)$-channel. However, the Yukawa couplings of the leptoquarks with the first generation of quarks and leptons are severely constrained from the atomic parity violation experiments [45], making this contribution small. In order to generate the model files for further simulation, we have incorporated the new physics Lagrangian in FEYNRULES (v2.3.13) [56,57] and subsequently generated model files are used to compute particle spectrum, relevant branching ratios and cross sections via MADGRAPH5 (v2.4.3) [58,59] with NNPDF23lo1 $[60,61]$ parton distribution function (PDF). We have used dynamic definition [62] of the factorization scale for the PDF and renormalization scale for evaluating the QCD coupling. Being mainly QCD driven, the pair production of leptoquarks [63] gets significant corrections from the higher order processes. In Ref. [64], next-to-leading (NLO) order in QCD $k$-factor for leptoquark pair production at the $13 \mathrm{TeV}$ LHC is estimated to be $\sim 1.4$. In our calculation, we have included the $k$-factor. The QCD pair production cross section of the leptoquarks $\left(\sigma\left(p p \rightarrow \Delta^{+2 / 3} \Delta^{-2 / 3}\right)\right)$ as a function of the leptoquark mass $\left(M_{\Delta^{ \pm 2 / 3}}\right)$ at the LHC with $13 \mathrm{TeV}$ center-of-mass energy is presented in Fig. 1. Being strongly interacting, leptoquark pair production cross sections are rather large at the LHC as it can be seen from Fig. 1. The pair production cross section varies from few picobarn

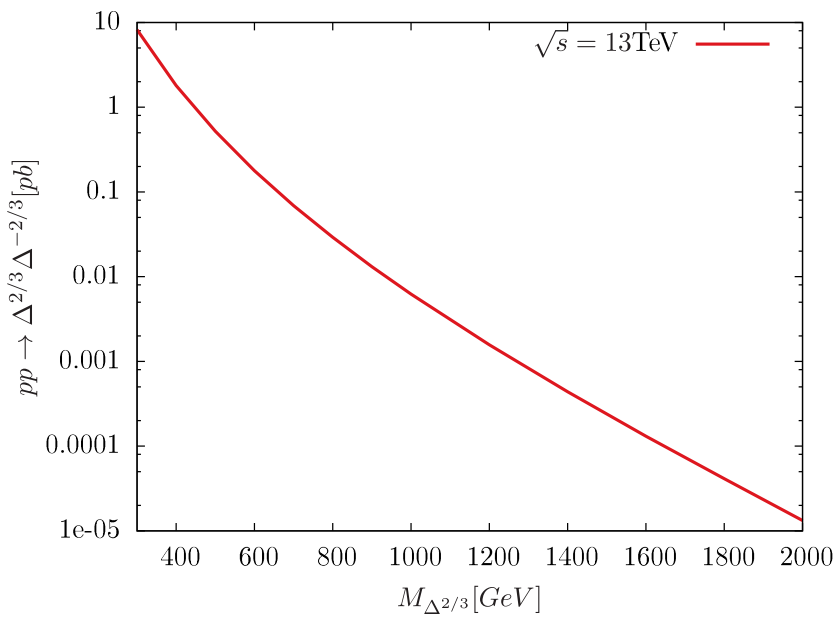

FIG. 1. Pair production cross section of leptoquarks at the LHC with $13 \mathrm{TeV}$ center-of-mass energy as a function of the leptoquark mass. to few femtobarn for the $\mathrm{LQ}$ mass between few hundred $\mathrm{GeV}$ to $\mathrm{TeV}$.

After being produced, leptoquarks decay into a quarklepton pairs via the Yukawa interactions of Eq. (4). For example, $\Delta^{ \pm 2 / 3}$ can decay into a down-type quark and charged lepton pair or a up-type quark and right-handed neutrino pair. The decay of $\Delta^{ \pm 2 / 3}$ into a down-type quark and charged lepton pair is proportional to $Y_{L}$ whereas, the decay into a up-type quark and right-handed neutrino pair is proportional to $Y_{R}$. For simplicity, we have assumed that leptoquark Yukawa matrices are diagonal and leptoquarks can dominantly couple to the third generation of SM fermions. ${ }^{3}$ Similarly, as mentioned earlier, we have also chosen the neutrino Yukawa matrix $\left(Y_{\nu}\right)$ to be diagonal in order to avoid constraints arising from nonobservation of lepton flavor violating decays. As a result of this choice the third generation RH neutrino (which will be denoted as $N_{\tau}$ in the mass basis) can only directly decay into a tau and $W$ boson or a light $\nu_{\tau}$ and $\mathrm{Z}$ (with smaller branching ratio) through its left-handed components which compels us to look for tau-enriched final states. This will also help us to reduce SM backgrounds, at least for the signal reagions we will be dealing with. We note in passing that keeping the flavor diagonal structure for $Y_{L, R}$, one may obtain $\mu$ rich final states for larger values of $Y_{R}^{22}\left(Y_{R}^{22} \gg Y_{L}^{22}\right.$ and $\left.Y_{L, R}^{22} \gg Y_{L, R}^{33}\right)$, but then one may face relatively difficult task in regard to $c$-jet tagging.

Thus, in this framework, $\Delta^{+2 / 3}$ dominantly decays into a top and third generation right-handed neutrino pair $\left(t N_{\tau}\right)$ or a bottom quark and tau lepton pair $(b \bar{\tau})$ depending on the relative values of $Y_{R}^{33}$ and $Y_{L}^{33}$, respectively. In Fig. 2, we have presented the branching ratio of $\Delta^{ \pm 2 / 3}$ into $t N_{\tau}$ and $b \tau$ pairs as a function of $Y_{R}^{33}$ for different values of $Y_{L}^{33}$, and write down the analytic expression of the decay widths in the appendix.

Fig. 2 clearly shows that $t N_{\tau}$ decay mode dominates for larger values of $Y_{R}^{33}$ compared to $Y_{L}^{33}$. The off-shell decays for example $\Delta^{2 / 3} \rightarrow \Delta^{1 / 3} W^{*}$ does not occur in this scenario due to mass degeneracy of the two LQs. In order to enhance the production of right-handed neutrinos from the decay of $\Delta^{ \pm 2 / 3}$, smaller $Y_{L}^{33}$ compared to $Y_{R}^{33}$ is preferred. Note that, for larger $Y_{L}^{33}$, the bounds on $\Delta^{ \pm 2 / 3}$ mass are much stronger from resonance searches in the lepton-jet invariant mass distribution at the LHC [65]. Therefore, in our analysis, we consider $Y_{R}^{33}>Y_{L}^{33}$ hence, $\Delta^{ \pm 2 / 3}$ dominantly decays into $t N_{\tau}$ pairs with almost $100 \%$ branching ratios.

In addition to the variation w.r.t Yukawa, we also show the variation w.r.t the RH neutrino mass. In Fig. 3, we show

\footnotetext{
${ }^{3}$ This choice is motivated as leptoquark decaying into a top quark and heavy neutrino results in higher jet, lepton multiplicities and larger missing energy in the final state.
} 

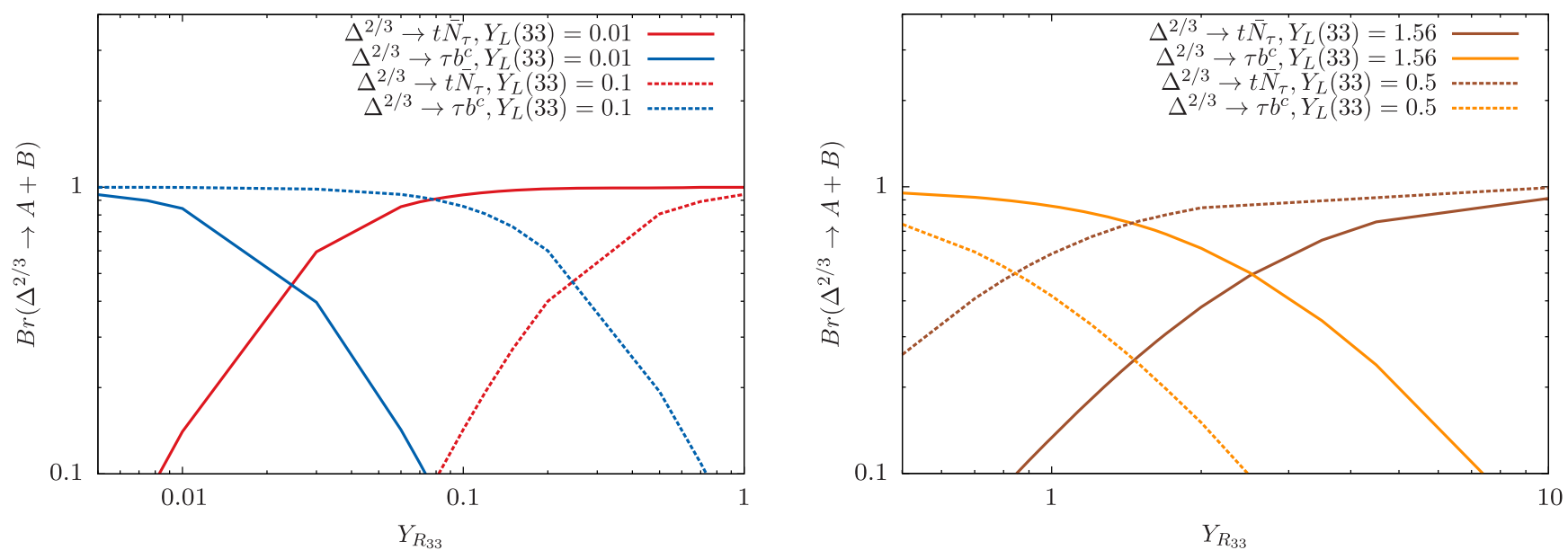

FIG. 2. Branching ratios of $\Delta^{ \pm 2 / 3}$ into $t N_{\tau}$ and $b \tau$ pairs as a function of $Y_{R}^{33}$ for different values of $Y_{L}^{33}$. Left and right panel correspond to $850 \mathrm{GeV}$ and $1 \mathrm{TeV}$ leptoquark masses, as illustrative examples.

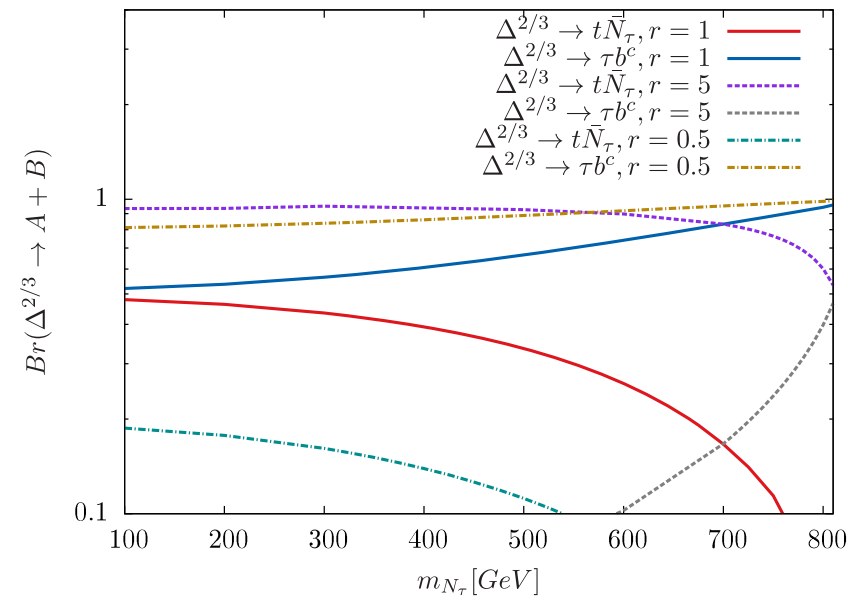

FIG. 3. Variation of branching ratios of $\Delta^{ \pm 2 / 3}$ into $t N_{\tau}$ and $b \tau$ pairs as a function of heavy neutrino mass $m_{N_{\tau}}$. The LQ mass is set to $1 \mathrm{TeV}$ and $r=\frac{Y_{R_{33}}}{Y_{L_{33}}}$.

the variation of the branching ratios of $\Delta^{2 / 3}$ with the RH neutrino mass $m_{N_{\tau}}$. The LQ mass is set to $1 \mathrm{TeV}$ and we define $r$ as the ratio of $\frac{Y_{R_{33}}}{Y_{L_{33}}}$ to delineate the dependence of the branching fraction on the $m_{N_{\tau}}$. Clearly, $\operatorname{Br}\left(\Delta^{2 / 3} \rightarrow\right.$ $t N_{\tau}$ ) would be the most dominant channel for $r>1$. For representation purpose we assume $r=5$, and we observe that the $\operatorname{Br}\left(\Delta^{2 / 3} \rightarrow t N_{\tau}\right)$ is always $100 \%$ if phase space is allowed. The fall in $\operatorname{Br}\left(\Delta^{2 / 3} \rightarrow t N_{\tau}\right)$ occurs near the kinematic threashold $m_{N_{\tau}} \sim 827 \mathrm{GeV}$ for the LQ mass $1 \mathrm{TeV}$. After the kinematic threshold, additional three body modes such as, $\Delta^{2 / 3} \rightarrow t W l, t Z \nu$ with branching ratio 30 $40 \%$ also open up. In our subsequent analysis, we work with higher $Y_{R}^{33}$, and relatively smaller mass of $N_{\tau}$ as compared to the LQ, so that the two body decay mode $\Delta^{ \pm 2 / 3} \rightarrow t N_{\tau}$ is almost $100 \%$.
The produced $N_{\tau}$ subsequently decays into a $\tau W$ pair or a $\nu_{\tau} Z^{4}$ pair. Therefore, the pair production of $\Delta^{ \pm 2 / 3}$ at the LHC gives rise to multiple top quarks, tau leptons, and $W$ and/or $Z$ bosons in the final state. Subsequent decays of those heavier SM particles result into multiple leptons, jets and missing transverse energy $\left(\mathscr{E}_{T}^{\prime}\right)$ signature at the LHC. Before going into the details of signal and background analysis, let us have a brief discussion on the present status of the heavy neutrino and leptoquark searches in collider and other experiments which lead to our choices of benchmark points for subsequent collider study.

\section{PRESENT STATUS, CONSTRAINTS AND CHOICE OF BENCHMARK POINTS}

The collider signatures of right-handed neutrinos have been extensively studied in the literature [33,43,44,66-75]. Usually, the search for a heavy Majorana neutrino is driven by lepton number violating final states which provide a smoking-gun signals for such scenarios. However, within the framework of inverse seesaw, the lepton number is broken by the $\mu$ parameter which is extremely small and, therefore, the usual same-sign dilepton signal is expected to be much weaker in this scenario compared to a trilepton signal [39]. In fact, the smallness of $\mu$ results in suppression of any lepton number violating (LNV) processes in this framework. Thus in the considered scenario, the firstgeneration Dirac neutrino Yukawa coupling $Y_{\nu}^{11}$, which is highly constrained for type-I seesaw mechanism from nonobservation of neutrinoless double beta decay $(0 \nu \beta \beta)$ [38], remains practically unconstrained. The experimental collaborations have put constraints on the heavy Majorana

\footnotetext{
${ }^{4}$ Though the decay widths of $N_{\tau}$ into $\tau W$ or $\nu_{\tau} Z$ are suppressed by the small left-handed and right-handed neutrino mixing angle, but in absence of any other decay modes, $N_{\tau}$ decays into $\tau W$ and $\nu_{\tau} Z$ pair with $67 \%$ and $33 \%$ branching ratios, respectively.
} 
neutrino masses depending on its choice of Dirac neutrino Yukawa couplings from the search of same flavor opposite-sign dilepton final state [76-81]. In the inverse seesaw scenario, these constraints are trivially satisfied. Comparatively, some other final states with various lepton-jet multiplicities can provide a more stringent constraint on the inverse seesaw scenario that can probe a mixing angle up to $\sim 10^{-2}$ for a heavy neutrino mass around $200 \mathrm{GeV}$ at $14 \mathrm{TeV}$ run of the LHC with a luminosity around $1000 \mathrm{fb}^{-1}$ [39]. In this work, we aim to probe up to a much higher RH neutrino mass range. This is achievable, as our RH neutrino production is not limited by the active-sterile mixing which is further constrained from precision studies [17,82-85]. Instead, the heavy neutrino is produced from leptoquark decays, that has strong interaction. This helps to obtain a relatively larger RH neutrino production cross section. Another potential source of the constraints on the neutrino sector may arise from the lepton flavor violating (LFV) decays that arise due to nondiagonal $Y_{\nu}$ or $M_{R}$. In this analysis, we consider these two matrices strictly diagonal which leads to vanishing contribution of the new physics contribution to the LFV decays.

Experimental collaborations have also searched for any possible hints for scalar leptoquarks at the LHC [86,87]. The favored final state to look for these exotic particles has been $\ell \ell j j$, where, $\ell=e, \mu, \tau$. The nonobservation of any new physics events have ruled out LQ up to masses as large as $1100 \mathrm{GeV}$. However, these existing limits are subjected to a particular choice of $Y_{L}$ that forces the leptoquarks to decay dominantly into a charged lepton and a light (or bottom) quark. In this work, however, we intend to explore a different coupling of the LQ, namely, $Y_{R}$ which forces the
LQ to decay via the heavy neutrinos. Therefore, taking a clue from the obtained branching ratio distribution of the LQs as shown in Fig. 2, we keep $Y_{L}$ smaller in comparison to $Y_{R}$ for all our benchmark points so that relatively lighter LQ masses can be probed which are still allowed by the LHC. However, the leptoquark decaying via heavy neutrinos can give rise to the same kind of multilepton signals which has usually been studied in the context of supersymmetric searches [88-90]. So far all such search results have turned out to be consistent with the SM predictions and hence these results can constrain the LQ as well as heavy neutrino masses in our scenario.

As has been mentioned, in this work, we have considered the pair production of $\Delta^{ \pm 2 / 3}$ and its subsequent decay into a top and a heavy neutrino. Hence, the collider phenomenological aspects of our study are determined by five parameters namely, leptoquark mass $\left(m_{\Delta^{ \pm 2 / 3}}\right)$, its couplings $Y_{L, R}^{33}$, right-handed neutrino mass $\left(m_{N_{\tau}}\right)$ and its coupling $m_{D}^{33}$. The pair-production cross section $\sigma\left(\Delta^{ \pm 2 / 3} \Delta^{\mp 2 / 3}\right)$ is determined by the choice of $m_{\Delta^{ \pm 2 / 3}}$ and its couplings. While, relative strength of $Y_{R}^{33}$ and $Y_{L}^{33}$ determines the decay of $\Delta^{ \pm 2 / 3}$ into right-handed neutrino $\left(N_{\tau}\right)$, the mass splitting between $\Delta^{ \pm 2 / 3}-N_{\tau}$ determines the shape of characteristic signal distributions. In order to present the numerical results of our analysis, we have chosen three benchmark points (BP). The relevant parameters for the collider phenomenology for those three BPs are listed in Tables II and III. We also present a bench-mark point (BP2 in Table III) which can potentially explain $B$-physics anomalies as mentioned earlier. In order to get a large $\operatorname{BR}\left(\Delta^{ \pm 2 / 3} \rightarrow t N_{\tau}\right)$, only large values of $Y_{R}^{33}$ are allowed which nearly saturates the perturbative limits $\sim 4 \pi$ even at

TABLE II. $m_{D}$ and $M_{R}$ taken as inputs, $\mu_{X}$ is the resulting matrix derived such that the oscillation parameters are in agreement.

\begin{tabular}{|c|c|c|c|c|c|c|c|c|c|}
\hline Parameters & \multicolumn{3}{|c|}{ BP1 } & \multicolumn{3}{|c|}{ BP2 } & \multicolumn{3}{|c|}{ BP3 } \\
\hline$m_{D}^{i i}$ & \multicolumn{3}{|c|}{$(0.1,0.1,0.1)$} & \multicolumn{3}{|c|}{$\left(10^{-9}, 10^{-9}, 0.1\right)$} & \multicolumn{3}{|c|}{$(0.1,0.1,0.1)$} \\
\hline$M_{R}^{i i}(\mathrm{GeV})$ & \multicolumn{3}{|c|}{$(1000.0,1000.0,400.0)$} & \multicolumn{3}{|c|}{$\left(10^{-6}, 10^{-6}, 600\right)$} & \multicolumn{3}{|c|}{$(1000.0,1000.0,800.0)$} \\
\hline$\mu_{X}(\mathrm{eV})$ & $\left(\begin{array}{c}9.233 \\
15.141 \\
2.799\end{array}\right.$ & $\begin{array}{l}15.141 \\
52.874 \\
22.228\end{array}$ & $\left.\begin{array}{c}2.799 \\
22.228 \\
15.921\end{array}\right)$ & $\left(\begin{array}{l}0.144 \\
0.237 \\
0.525\end{array}\right.$ & $\begin{array}{l}0.237 \\
0.826 \\
4.168\end{array}$ & $\left.\begin{array}{c}0.525 \\
4.168 \\
35.821\end{array}\right)$ & $\left(\begin{array}{c}14.427 \\
23.658 \\
6.998\end{array}\right.$ & $\begin{array}{l}23.658 \\
82.616 \\
55.569\end{array}$ & $\left.\begin{array}{c}6.998 \\
55.569 \\
63.683\end{array}\right)$ \\
\hline Resulting PMNS & $\left(\begin{array}{l}0.810 \\
0.567 \\
0.148\end{array}\right.$ & $\begin{array}{l}0.507 \\
0.550 \\
0.663\end{array}$ & $\left.\begin{array}{l}0.295 \\
0.612 \\
0.733\end{array}\right)$ & $\left(\begin{array}{l}0.806 \\
0.548 \\
0.143\end{array}\right.$ & $\begin{array}{l}0.493 \\
0.556 \\
0.647\end{array}$ & $\left.\begin{array}{l}0.284 \\
0.610 \\
0.740\end{array}\right)$ & $\left(\begin{array}{l}0.806 \\
0.573 \\
0.148\end{array}\right.$ & $\begin{array}{l}0.511 \\
0.549 \\
0.661\end{array}$ & $\left.\begin{array}{l}0.297 \\
0.608 \\
0.736\end{array}\right)$ \\
\hline
\end{tabular}

TABLE III. Relevant parameters and production cross section (including the k-factor) of leptoquarks at LHC, for 13 TeV c.m. energy.

\begin{tabular}{lccccc}
\hline \hline Benchmarks & $m_{\Delta^{(2 / 3)}}(\mathrm{GeV})$ & $m_{N_{\tau}}(\mathrm{GeV})$ & $Y_{L}{ }^{i i}$ & $Y_{R}{ }^{i i}$ & $\sigma_{\Delta^{(2 / 3)} \bar{\Delta}^{(2 / 3)}(\mathrm{fb})}$ \\
\hline BP1 & 850.0 & 400.0 & $\left(0.0,10^{-3}, 10^{-3}\right)$ & $\left(0.0,10^{-3}, 0.1\right)$ & 18.760 \\
BP2 & 1000.0 & 600.0 & $\left(0.0,10^{-3}, 1.5\right)$ & $\left(0.0,10^{-3}, 12.56\right)$ & 6.342 \\
BP3 & 1200.0 & 800.0 & $\left(0.0,10^{-3}, 10^{-3}\right)$ & $\left(0.0,10^{-3}, 0.1\right)$ & 1.512 \\
\hline \hline
\end{tabular}


the $\mathrm{TeV}$ scale. However, we recall that LQs are always associated with larger symmetry. Assuming a larger gauge group at the $\mathrm{TeV}$ scale can potentially help to avoid Landau-pole problem due to large value of $Y_{R}^{33}$.

Table II shows the choices of the neutrino sector parameters, $m_{D}$ and $M_{R}$ and the resulting $\mu_{X}$ after fitting the neutrino oscillation data as mentioned in Sec. II A. We have also presented the obtained light $3 \times 3$ neutrino mass matrices which are in good agreement with the allowed PMNS matrix elements. Nondiagonal structure of $\mu_{X}$ reflects the fact that we have assumed diagonal $Y_{\nu}^{i i}$, hence $m_{D}^{i i}$, to suppress lepton flavor violating observables. In the present context, the effective production cross section of the right-handed neutrinos have been considerably larger, thanks to larger productions for LQs $\left(\Delta^{(2 / 3)}\right)$ and their decays to $t N_{\tau}$ with almost $100 \%$ branching ratio. Table III shows our choices of LQ $\left(\Delta^{(2 / 3)}\right)$ masses and their relevant couplings along with their pair production cross section for the three benchmark points. We now proceed to discuss our collider analysis of the leptoquarks decaying via heavy neutrinos yielding various possible novel signal regions.

\section{COLLIDER ANALYSIS AND RESULTS}

As has already been discussed, our primary focus is on the interaction induced by the Yukawa couplings of righthanded neutrinos with the leptoquarks which give rise to interesting signals at the LHC. Leptoquarks, being strongly interacting, are copiously produced at hadron colliders. Therefore, in the framework of leptoquark model, the production of right-handed neutrinos could be enhanced significantly. Moreover, the decay of leptoquarks are usually accompanied by hard jets and/or leptons which could enhance the efficiency of signal selection criteria to reduce the SM background. With the LHC running at its near kinematic threshold, at $\sqrt{s}=13 \mathrm{TeV}$, one also has to deal with the large hadronic backgrounds while looking for any new physics signal. One way to avoid this menace is to look for more and more lepton enriched final states which naturally tend to reduce these unwanted background contributions. We have, therefore, focussed to identify the signal regions in a way so that the maximum number of hard leptons, like-sign or otherwise, are identified in the final state while not tagging all the $b$ jets and $\tau$ jets arising from the cascades in order to maximize the signal rates. In the framework of the present model, different signal regions (SR) can be defined with multiple leptons when $\Delta^{ \pm 2 / 3} \rightarrow$ $t N_{\tau}$ and $N_{\tau} \rightarrow \tau W$ or $\nu_{\tau} Z$ are followed by the leptonic decay of both the top quarks and the gauge bosons present in the cascade:

$$
\begin{aligned}
p p & \rightarrow \Delta^{ \pm 2 / 3} \Delta^{\mp 2 / 3} \rightarrow\left(t \bar{N}_{\tau}\right)\left(\bar{t} N_{\tau}\right) \\
& \rightarrow b \bar{b} W^{+} W^{-} \tau^{+} \tau^{-} W^{+} W^{-} \\
& \rightarrow b \bar{b} W^{+} W^{-} \nu_{\tau} \bar{\nu}_{\tau} Z Z \\
& \rightarrow b \bar{b} W^{+} W^{-} \tau^{+} \nu_{\tau} W^{-} Z .
\end{aligned}
$$

The above three possible decay chains can potentially give rise to maximum six leptons in the final state. Any final state with such a large lepton multiplicity will be a very clean signal devoid of SM background. However, that will require both the $N_{\tau}$ s to decay via the $Z$ boson which has a suppressed branching ratio compared to its $W$-decay mode. Moreover, the $Z$-boson decay branching ratios to the electrons and muons are also suppressed. Hence we restrict ourselves to signal regions with at least three or four leptons in the final state. Note that, here we focus on the RH neutrino decay to $\tau$ lepton. The $e, \mu$ states are mostly generated from the gauge boson decays. Another interesting signal region (SR) can be constructed from the first and third cascade decays shown in Eq. (10) when two of the same-sign $W$ bosons decay leptonically and the other $W$ and (or) $Z$ bosons decay hadronically. This will result into a same-sign dilepton (SSD) signal along with multiple jets and $E_{T}$. Such a SSD signal is expected to be different from the usual heavy neutrino signals both in terms of jet multiplicity and $E_{T}$ distribution.

For a detailed collider simulation, we have generated parton level events using MADGRAPH5 and subsequently passed the events into PYTHIA (v6.4.28) [91] for simulating initial state radiation/final state radiation (ISR/FSR), decay of the heavy particles and hadronization of the final state quarks. In order to reconstruct the physics objects like, jets, leptons, photons and missing transverse energy, we have used the fast detector simulator DeLPHES-v3.3.3 [92-94]. The jets are reconstructed by anti- $k_{t}$ algorithm [95] implemented in the FASTJET package [95-97] with a cone of $\Delta R=0.4$ and minimum transverse momentum of $20 \mathrm{GeV}$. The tagging and mistagging efficiencies of $b$ jet and $\tau$ jet have been incorporated according to the latest ATLAS studies in this regard [98] in DeLPHES3. After the object reconstruction, leptons with $p_{T}^{l}>10 \mathrm{GeV}$ and $\left|\eta^{l}\right|<2.5$ and jets with $p_{T}^{j}>20 \mathrm{GeV}$ and $\left|\eta^{j}\right|<2.5$ are considered for the further event selection. Furthermore, the electrons and muons satisfying the $p_{T}^{l}$ criteria have been selected with $95 \%$ and $85 \%$ efficiencies for $\left|\eta^{l}\right|<1.5$ and $1.5<\left|\eta^{l}\right|<2.5$ respectively. Finally, the $b$ and $\tau$ jets are tagged with $p_{T}>20 \mathrm{GeV}$ while the rest of the jets are tagged with different $p_{T}$ requirements of our signal regions following the ATLAS event selection criteria as in Refs. [89,90].

Multiple sources of missing energy throughout the cascade and the large multiplicity of both jets and(or) leptons are likely to produce hard $E_{T}$ and $M_{E F F}$ distributions, where $M_{E F F}=\sum_{i} p_{T_{i}}^{j}+\sum_{i} p_{T_{i}}^{\ell}+\mathscr{E}_{T}^{\dagger}$. In Fig. 4, we have shown these kinematic distributions for the three benchmark points introduced in Sec. III.

The distributions in Fig. 4 are obtained for final states containing at least two leptons. The blue, red and black lines represent $\mathrm{BP} 1, \mathrm{BP} 2$ and $\mathrm{BP} 3$, respectively. As expected, the hardness of the distributions increases with increasing leptoquark and heavy neutrino masses. The final 

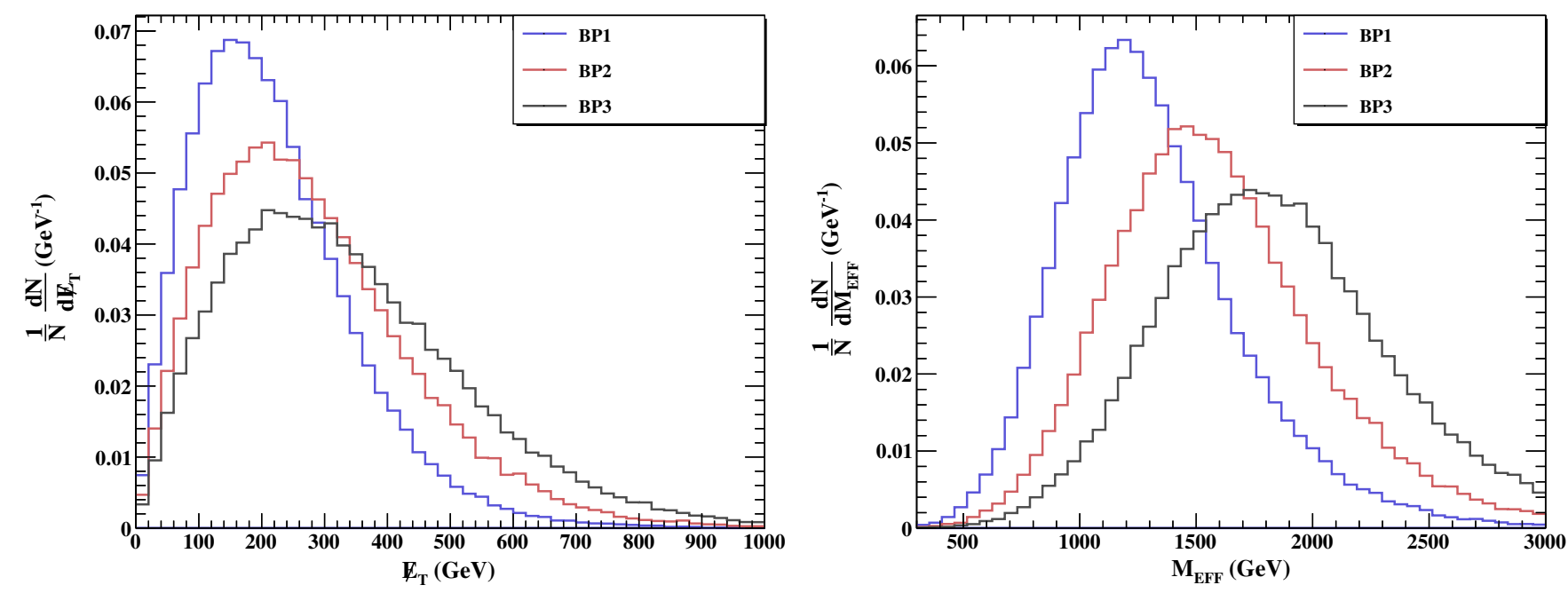

FIG. 4. Normalized distributions of $E_{T}$ and $M_{E F F}$ for final states consisting of at least two leptons corresponding to the three benchmark points.

state should also consist a large number of jets including multiple $b$ and $\tau$ jets. Tagging the $b / \tau$ jets can reduce the SM backgrounds significantly. The large QCD background at the LHC makes it difficult for any new physics signal with multiple jets to be observed. Tagging additional $b / \tau$ jets helps to reduce this background very effectively. Topquark associated production channels also give rise to large background contributions which can be minimized by demanding a $\tau$ jet in the final states. However, the detection efficiencies of $b / \tau$ jets vary with their $p_{T} \mathrm{~s}$ and hence tagging all the $b$ and (or) $\tau$ jets can also reduce the signal rates considerably. Taking into account all these factors, we define four SRs as shown in Table IV. We have categorized the choice of lepton and jet multiplicities and their $p_{T}$ requirement of the jets, $E_{T}$ and $M_{E F F}$ under $\mathrm{C} 0, \mathrm{C} 1$ and $\mathrm{C} 2$, respectively, as shown in the table.

For most of the signal regions focus on the large lepton and jet multiplicity and the hard $E_{T}$ and $M_{E F F}$ distributions to achieve a good signal to background ratio. Among the first three SRs, SR3Lb and SR3Lbt are expected to have some SM background contributions while SR4Lbt is likely to be a much cleaner channel. As mentioned before, any lepton number violating signals are suppressed in the framework of the inverse seesaw scenario due to the smallness of $\mu_{X}$. Hence the usual SSD signal is not viable to probe for inverse seesaw extended SM. However, here one can obtain a different kind of same-sign dilepton signal as shown in SRSS2Lb, where the same-sign criteria is imposed on the events with exactly two leptons in the final state. Such a signal region can, therefore, be a distinguishing feature for such a scenario. The choice of our signal regions are motivated from some experimental studies with final states consisting of multiple jets and leptons in the context of supersymmetry [88-90]. There are multiple signal regions studied in these works, which can be relevant to our scenario. Therefore, we have checked the consistency of our chosen benchmark points with these experimental results using CheckMATE $[99,100]$ which determines whether a chosen model parameter space is excluded or not at 95\% confidence level [101] by comparing it to the relevant experimental analyses. ${ }^{5}$

The obtained signal cross sections at $13 \mathrm{TeV}$ center-ofmass energy are presented in Table $\mathrm{V}$ with cut-flow numbers for the signal regions and cuts $\mathrm{C} 0-\mathrm{C} 2$ as defined in Table IV corresponding to the three BPs defined in Table III. Irrespective of the choice of different signal regions, the $M_{E F F}$ cut (C2) does not affect the signal cross sections for any of the benchmark points. This is quite expected as Fig. 4 clearly shows our choice of the $E_{T}$ cut (C1) automatically pushes the phase space to a hard kinematic region where the $M_{E F F}$ cut is trivially satisfied. However, C2 is very useful to reduce the SM backgrounds for these signal regions as mentioned in $[89,90]$.

(i) The signal region SR3Lb with three or more leptons and at least one tagged $b$ jet has also been studied by the ATLAS Collaboration although in a different context [89]. They estimated a combined SM background cross section of $0.56 \mathrm{fb}$. In absence of any significant deviation from the SM background prediction, the ATLAS Collaboration has derived an upper bound of $0.41 \mathrm{fb}$ on new physics trilepton cross section in SR3Lb signal region. As the numbers in Table $\mathrm{V}$ suggest, all our benchmark points are well within this experimental bound. We have also checked that the ATLAS study of SR3Lb imposes a lower bound of about $700 \mathrm{GeV}$ on the mass of the 3rd generation leptoquark if it

\footnotetext{
${ }^{5}$ The analyses of Ref. [89] have not yet been included in CheckMATE. We have checked consistency of our benchmark points with these results using our own code.
} 
TABLE IV. The four different signal regions with lepton, jet multiplicities and kinematic cuts.

\begin{tabular}{|c|c|c|c|c|c|c|c|c|}
\hline \multirow[b]{2}{*}{ Signal region } & \multicolumn{6}{|c|}{$\mathrm{C} 0$} & \multirow{2}{*}{$\frac{\mathrm{C} 1}{E_{T}^{\prime}(\mathrm{GeV})}$} & \multirow{2}{*}{$\frac{\mathrm{C} 2}{M_{E F F}(\mathrm{GeV})}$} \\
\hline & $N_{\ell}$ & $N_{j}$ & $N_{b}$ & $N_{\tau}$ & $p_{T}^{j}(\mathrm{GeV})$ & $p_{T}^{b / \tau}(\mathrm{GeV})$ & & \\
\hline SR3Lb & $\geq 3$ & $\geq 4$ & $\geq 1$ & $\ldots$ & $>40$ & $>20$ & $>200$ & $>600$ \\
\hline SR3Lbt & $\geq 3$ & $\geq 4$ & $\geq 1$ & $\geq 1$ & $>40$ & $>20$ & $>200$ & $>600$ \\
\hline SR4Lbt & $\geq 4$ & $\ldots$ & $\geq 1$ & $\geq 1$ & $>40$ & $>20$ & $>200$ & $>600$ \\
\hline SRSS2Lb & $\geq 2$ & $\geq 4$ & $\geq 1$ & $\ldots$ & $>50$ & $>20$ & $>150$ & $\geq 550$ \\
\hline
\end{tabular}

TABLE V. Cut-flow table for signal cross sections with the cuts mentioned in Table IV for the different SRs and BPs at the LHC at $13 \mathrm{TeV}$ center-of-mass energy.

\begin{tabular}{|c|c|c|c|c|c|c|c|c|c|}
\hline \multirow[b]{3}{*}{ Signal region } & \multicolumn{9}{|c|}{ Signal cross section $(\mathrm{fb})$} \\
\hline & \multicolumn{3}{|c|}{ BP1 } & \multicolumn{3}{|c|}{$\mathrm{BP} 2$} & \multicolumn{3}{|c|}{ BP3 } \\
\hline & $\mathrm{C} 0$ & $\mathrm{C} 1$ & $\mathrm{C} 2$ & $\mathrm{C} 0$ & $\mathrm{C} 1$ & $\mathrm{C} 2$ & $\mathrm{C} 0$ & $\mathrm{C} 1$ & $\mathrm{C} 2$ \\
\hline SR3Lb & 0.376 & 0.181 & 0.181 & 0.130 & 0.081 & 0.081 & 0.330 & 0.024 & 0.024 \\
\hline SR3Lbt & 0.137 & 0.062 & 0.062 & 0.047 & 0.027 & 0.027 & 0.012 & 0.009 & 0.009 \\
\hline SR4Lbt & 0.016 & 0.007 & 0.007 & 0.006 & 0.003 & 0.003 & 0.002 & 0.001 & 0.001 \\
\hline SRSS2Lb & 0.924 & 0.598 & 0.598 & 0.320 & 0.245 & 0.245 & 0.080 & 0.066 & 0.066 \\
\hline
\end{tabular}

dominantly decays into a top-quark and a heavy neutrino in the framework of the present model. Taking into account the SM background contribution, one can obtain a statistical significance ${ }^{6}$ of $3 \sigma$ for BP1 at an integrated luminosity $(L)$ of $\sim 200 \mathrm{fb}^{-1}$. For $\mathrm{BP}$ 2, with higher LQ mass, in order to achieve similar statistical significance, one needs $L \sim 900 \mathrm{fb}^{-1}$. BP3 is unlikely to be probed in this SR even at a relatively high luminosity of $L \sim 3000 \mathrm{fb}^{-1}$.

(ii) Demanding at least one additional $\tau$ jet (i.e., the SR SR3Lbt) reduces the signal cross section to $0.062 \mathrm{fb}$ for BP1. However, demanding an extra $\tau$-tagged jet on the top of SR3Lb reduces the SM background cross section at least by a factor of $\alpha_{W}$. Thus, even the $\sim 12$ events expected at $L \sim 200 \mathrm{fb}^{-1}$ for BP1 is relatively background free and can act as a complementary channel to SR3Lb. This SR can be more economical for BP2 and BP3 compared to the previous one. As the numbers in Table $\mathrm{V}$ suggest, both these benchmark points can be probed below and around $L=1000 \mathrm{fb}^{-1}$, respectively.

(iii) SR4Lbt is even a more cleaner channel to probe for the present scenario. However, as the numbers suggest, in order to get some signal events $\left(N_{\text {sig }} \sim 7\right.$ for BP1) in this signal region, one has to go for high luminosity $L \sim 1000 \mathrm{fb}^{-1}$. Thus this signal region can only serve as a complementary channel to the previous two if any excess of events are found in

\footnotetext{
${ }^{6}$ Statistical significance, $\mathcal{S}=\frac{s}{\sqrt{s+b}}$, where $s=$ number of signal events and $b=$ number of background events.
}

either of them. For LQ masses around $1000 \mathrm{GeV}$ and above, as in BP2 and BP3, this SR does not look promising enough due to extremely small signal cross section.

(iv) The signal region SRSS2Lb has been studied in the context of supersymmetry at the LHC. Here we have used the same set of selection criteria and kinematic cuts as the ATLAS Collaboration [90] to obtain the signal cross section. The experimental upper limit on new physics cross section in this signal region remains at $2.8 \mathrm{fb}$ which is much larger than that obtained for any of the benchmark points of our choice. Although the SM background contribution in this channel is expected to be the largest compared to the other SRs, the large signal cross section makes it the most promising channel to probe at the LHC. The ATLAS Collaboration quoted the total SM background contribution as $1.406 \mathrm{fb}$ [90]. This leads to a statistical significance of $3 \sigma$ at $L \sim 55 \mathrm{fb}^{-1}$ for BP1 which means this parameter space can immediately be probed with the accumulated data at the LHC at present. To achieve similar statistical significance for BP2 and BP3 one requires $L \sim 250 \mathrm{fb}^{-1}$ and $3000 \mathrm{fb}^{-1}$, respectively.

From the above, it is evident that a lighter LQ mass and lighter RH neutrino, such as $M_{\Delta^{2 / 3}}=850, m_{N_{\tau}}=400 \mathrm{GeV}$ can be probed in very near future with the data already accumulated at the LHC through at least two same-sign leptons, multijet and one tagged $b$ jet in the final state. Even a heavier neutrino $(\sim 600 \mathrm{GeV})$ can be probed in association with a $\sim 1000 \mathrm{GeV}$ leptoquark mass at a relatively 
lower luminosity of $250 \mathrm{fb}^{-1}$ in this final state. To probe higher mass ranges, as represented by BP3, the HL-LHC run is required. Among the other SRs, SR3Lb and SR3Lbt turn out to be the other viable options. However, they will be relevant only if some hint of new physics is obtained at lower luminosity via SRSS2Lb. Besides, it also provides us with a nice distinguishing feature that can be used to differentiate an usual inverse seesaw extended SM scenario from the present one. Note that, almost two third contribution of the signal cross section in this SR arises from same-sign two-lepton final state, a contribution which is expected to be negligible in the usual inverse seesaw extended SM.

\section{CONCLUSION AND OUTLOOK}

In this work, we have considered a minimal extension of the SM that can (i) produce neutrino masses and mixing angles and (ii) explain $B$-physics anomalies. We consider a scalar LQ with the hypercharge $Y=\frac{1}{6}$ and embed it in a inverse-seesaw framework with $\mathrm{TeV}$ scale or even lighter sterile neutrinos. Presence of a dominant flavor diagonal LQ- $\nu_{R}$ coupling can greatly enhance the effective production cross section of the heavy neutrino states in comparison to the canonical sterile neutrino production through charged/neutral current weak interactions. We have studied a rather unexplored decay mode of the leptoquark, i.e, to a top quark and heavy right-handed neutrino with almost $100 \% \mathrm{Br}$. This results in various multilepton signals associated with multiple jets (including $b$ and $\tau$ jets) and missing transverse energy. We have explored four such signal regions and carried out a comparative analysis aimed at probing heavy neutrino masses. We find that while a $\mathrm{RH}$ neutrino of mass $400 \mathrm{GeV}$ can immediately be probed at the LHC with at least one same-sign dilepton and multijet signal, a relatively heavier neutrino mass scales such as $800 \mathrm{GeV}$ can be probed in the high luminosity run of LHC. We have also observed that a mass bound of $700 \mathrm{GeV}$ on the LQ mass can be derived following the ATLAS $13 \mathrm{TeV}$ search in trilepton channel associated with multiple jets and at least one $b$-tagged jet provided the LQ decays dominantly into a top-quark and a heavy neutrino.

We have also presented a same-sign dilepton signal region which is expected to yield a much smaller event rate at the collider in the inverse seesaw extended SM. However, in the presence of leptoquarks where the heavy neutrinos are produced from the decay of these colored particles alongside top quarks, we have shown that one can expect significant event rates in this same-sign dilepton channel associated with multiple jets and missing energy.

Qualitatively all these can be considered as nice distinguishing features between the conventional inverse seesaw and the leptoquark associated inverse seesaw model. Quantatively, one can observe that much larger mass scale for heavy neutrinos can be reached through the aforesaid signal regions, specially via same-sign dileptons. Finally, allowing small off-diagonal structure in the LQ- $\nu_{R}$ coupling may lead to new avenues to LHC physics and flavor violations which requires a dedicated study.

\section{ACKNOWLEDGMENTS}

K. G. and M. M. acknowledge the support of a DST-INSPIRE FACULTY research grant. The work of S. M. is partially supported by funding available from the Department of Atomic Energy, Government of India, for the Regional Centre for Accelerator-based Particle Physics (RECAPP), Harish-Chandra Research Institute. S.M. acknowledges the hospitality of the Institute of Physics, Bhubaneswar, during the initial phase of the work.

\section{APPENDIX: RELEVANT DECAYS OF THE LEPTOQUARK}

Below, we write down the different two body decays of the LQ, relevant for our work.

$$
\begin{gathered}
\Gamma\left(\Delta^{2 / 3} \rightarrow t \bar{N}_{\tau}\right)=\frac{\left(Y_{R}^{33}\right)^{2}\left(\left(V_{N}^{53}\right)^{2}+\left(V_{N}^{63}\right)^{2}\right)}{16 \pi\left(m_{\Delta^{(2 / 3)}}\right)^{3}}\left(\left(m_{\Delta^{(2 / 3)}}\right)^{2}-m_{N_{\tau}}^{2}-m_{t}^{2}\right) \sqrt{\left(\left(m_{\Delta^{(2 / 3)}}\right)^{2}-\left(m_{N_{\tau}}+m_{t}\right)^{2}\right)\left(\left(m_{\Delta^{(2 / 3)}}\right)^{2}-\left(m_{N_{\tau}}-m_{t}\right)^{2}\right)} \\
\Gamma\left(\Delta^{2 / 3} \rightarrow b \bar{\tau}\right)=\frac{\left(Y_{L}^{33}\right)^{2}}{16 \pi\left(m_{\Delta^{(2 / 3)}}\right)^{3}}\left(\left(m_{\Delta^{(2 / 3)}}\right)^{2}-m_{b}^{2}-m_{\tau}^{2}\right) \sqrt{\left(\left(m_{\Delta^{(2 / 3)}}\right)^{2}-\left(m_{b}+m_{\tau}\right)^{2}\right)\left(\left(m_{\Delta^{(2 / 3)}}\right)^{2}-\left(m_{b}-m_{\tau}\right)^{2}\right)}
\end{gathered}
$$

where, $V_{N}^{i j}(i, j=1, \ldots, 6)$ diagonalizes the heavy neutrino mass matrix $\left(\mathcal{M}_{N}\right)$ written in the basis $\left\{\nu_{R}^{1}, \nu_{R}^{2}, \nu_{R}^{3}, X^{1}\right.$, $\left.X^{2}, X^{3}\right\}$. Note that all the six heavy neutrino masses are driven by the choices of $M_{R}^{i i}$ since all the diagonal entries of $\mathcal{M}_{N}$ are essentially zero $\left(\mu_{X} \ll M_{R}\right)$. Upon diagonalization of $\mathcal{M}_{N}$, we obtain three pairs of mass degenerate heavy neutrino states with masses $\simeq M_{R}^{11}, M_{R}^{22}$ and $M_{R}^{33}$, respectively. While writing $V_{N}$, the eigenvalues are arranged from heavy to light order. The fifth and sixth eigenvalues (mass degenerate) are driven by the choice of $M_{R}^{33}$ since by our choice, $M_{R}^{33}<M_{R}^{11}, M_{R}^{22}$. Only $V_{N}^{53}$ and $V_{N}^{63}$ elements are of importance for the decay $\Delta^{2 / 3} \rightarrow t \bar{N}_{\tau}$ since the relevant term in the Lagrangian is $Y_{R}^{i j} \bar{u}_{L}^{i} \nu_{R}^{j} \Delta^{(2 / 3)}$ and we have chosen to work with diagonal $Y_{R} . m_{t}, m_{b}$ and $m_{\tau}$ are the masses of top quark, bottom quark and $\tau$ lepton, respectively. 
[1] ATLAS experiment public results, https://twiki.cern.ch/ twiki/bin/view/AtlasPublic.

[2] CMS experiment public results, https://twiki.cern.ch/twiki/ bin/view/CMSPublic.

[3] M. C. Gonzalez-Garcia and M. Maltoni, Phys. Rep. 460, 1 (2008).

[4] M. C. Gonzalez-Garcia, M. Maltoni, and T. Schwetz, Nucl. Phys. B908, 199 (2016).

[5] P. Minkowski, Phys. Lett. 67B, 421 (1977).

[6] R. N. Mohapatra and G. Senjanovic, Phys. Rev. Lett. 44, 912 (1980).

[7] M. Gell-Mann, P. Ramond, and R. Slansky, Print-80-0576 (CERN).

[8] T. Yanagida, in Proceedings of the Workshop on the Baryon Number of the Universe and Unified Theories, Tsukuba, Japan, 1979 (National Lab for High Energy Physics, 1979), p. 109.

[9] S. L. Glashow, NATO Adv. Study Inst. Ser. B Phys. 59, 687 (1980).

[10] J. Schechter and J. W. F. Valle, Phys. Rev. D 25, 774 (1982).

[11] J. Schechter and J. W. F. Valle, Phys. Rev. D 22, 2227 (1980).

[12] S. Weinberg, Phys. Rev. Lett. 43, 1566 (1979).

[13] S. Weinberg, Phys. Rev. D 22, 1694 (1980).

[14] M. Magg and C. Wetterich, Phys. Lett. 94B, 61 (1980).

[15] T. P. Cheng and L.-F. Li, Phys. Rev. D 22, 2860 (1980).

[16] R. Foot, H. Lew, X. G. He, and G. C. Joshi, Z. Phys. C 44, 441 (1989).

[17] S. Antusch and O. Fischer, J. High Energy Phys. 05 (2015) 053.

[18] R. N. Mohapatra, Phys. Rev. Lett. 56, 561 (1986).

[19] S. Nandi and U. Sarkar, Phys. Rev. Lett. 56, 564 (1986).

[20] R. N. Mohapatra and J. W. F. Valle, Phys. Rev. D 34, 1642 (1986).

[21] G. 't Hooft, NATO Sci. Ser. B 59, 135 (1980).

[22] J. Cao, X. Guo, Y. He, L. Shang, and Y. Yue, J. High Energy Phys. 10 (2017) 044.

[23] B. Karmakar and A. Sil, Phys. Rev. D 96, 015007 (2017).

[24] R. Sinha, R. Samanta, and A. Ghosal, Phys. Lett. B 759, 206 (2016).

[25] E. Arganda, M. J. Herrero, X. Marcano, and C. Weiland, Phys. Rev. D 93, 055010 (2016).

[26] A. Abada, D. Das, A. Vicente, and C. Weiland, J. High Energy Phys. 09 (2012) 015.

[27] A. Abada, D. Das, and C. Weiland, J. High Energy Phys. 03 (2012) 100.

[28] A. Abada, G. Bhattacharyya, D. Das, and C. Weiland, Phys. Lett. B 700, 351 (2011).

[29] E. Arganda, M. J. Herrero, X. Marcano, and C. Weiland, Phys. Rev. D 91, 015001 (2015).

[30] E. Arganda, M. J. Herrero, X. Marcano, and C. Weiland, Phys. Lett. B 752, 46 (2016).

[31] V. De Romeri, M. J. Herrero, X. Marcano, and F. Scarcella, Phys. Rev. D 95, 075028 (2017).

[32] E. Arganda, M. Herrero, X. Marcano, R. Morales, and A. Szynkman, Phys. Rev. D 95, 095029 (2017).

[33] C.-Y. Chen and P.S. B. Dev, Phys. Rev. D 85, 093018 (2012).
[34] A. Das and N. Okada, Phys. Rev. D 88, 113001 (2013).

[35] P. Bandyopadhyay, E. J. Chun, H. Okada, and J.-C. Park, J. High Energy Phys. 01 (2013) 079.

[36] P. S. B. Dev, A. Pilaftsis, and U.-k. Yang, Phys. Rev. Lett. 112, 081801 (2014).

[37] A. Das, P. S. Bhupal Dev, and N. Okada, Phys. Lett. B 735, 364 (2014).

[38] F. F. Deppisch, P. S. Bhupal Dev, and A. Pilaftsis, New J. Phys. 17, 075019 (2015).

[39] A. Das and N. Okada, Phys. Rev. D 93, 033003 (2016).

[40] S. Mondal and S. K. Rai, Phys. Rev. D 94, 033008 (2016).

[41] S. Banerjee, P. S. B. Dev, A. Ibarra, T. Mandal, and M. Mitra, Phys. Rev. D 92, 075002 (2015).

[42] A. Das, arXiv:1701.04946.

[43] D. Alva, T. Han, and R. Ruiz, J. High Energy Phys. 02 (2015) 072.

[44] C. Degrande, O. Mattelaer, R. Ruiz, and J. Turner, Phys. Rev. D 94, 053002 (2016).

[45] I. Doršner, S. Fajfer, A. Greljo, J. F. Kamenik, and N. Košnik, Phys. Rep. 641, 1 (2016).

[46] J. C. Pati and A. Salam, Phys. Rev. D 10, 275 (1974); 11, 703(E) (1975).

[47] H. Georgi and S. L. Glashow, Phys. Rev. Lett. 32, 438 (1974).

[48] H. Georgi, AIP Conf. Proc. 23, 575 (1975).

[49] H. Fritzsch and P. Minkowski, Ann. Phys. (N.Y.) 93, 193 (1975).

[50] W. Altmannshofer, M. Carena, and A. Crivellin, Phys. Rev. D 94, 095026 (2016).

[51] D. Bečirević, S. Fajfer, N. Košnik, and O. Sumensari, Phys. Rev. D 94, 115021 (2016).

[52] D. Bečirević, S. Fajfer, and N. Košnik, Phys. Rev. D 92, 014016 (2015).

[53] I. Doršner, S. Fajfer, N. Košnik, and I. Nišandžić, J. High Energy Phys. 11 (2013) 084.

[54] A. Abada and M. Lucente, Nucl. Phys. B885, 651 (2014).

[55] O. Sumensari (private communication).

[56] N. D. Christensen and C. Duhr, Comput. Phys. Commun. 180, 1614 (2009).

[57] A. Alloul, N. D. Christensen, C. Degrande, C. Duhr, and B. Fuks, Comput. Phys. Commun. 185, 2250 (2014).

[58] J. Alwall, M. Herquet, F. Maltoni, O. Mattelaer, and T. Stelzer, J. High Energy Phys. 06 (2011) 128.

[59] J. Alwall, R. Frederix, S. Frixione, V. Hirschi, F. Maltoni, O. Mattelaer, H. S. Shao, T. Stelzer, P. Torrielli, and M. Zaro, J. High Energy Phys. 07 (2014) 079.

[60] R. D. Ball et al., Nucl. Phys. B867, 244 (2013).

[61] R. D. Ball et al. (NNPDF Collaboration), J. High Energy Phys. 04 (2015) 040.

[62] https://cp3.irmp.ucl.ac.be/projects/madgraph/wiki/FAQGeneral-13.

[63] B. Diaz, M. Schmaltz, and Y.-M. Zhong, J. High Energy Phys. 10 (2017) 097.

[64] T. Mandal, S. Mitra, and S. Seth, Phys. Rev. D 93, 035018 (2016).

[65] CMS Collaboration, Search for the third-generation scalar leptoquarks and heavy right-handed neutrinos in $\tau_{l} \tau_{h} j j$ final states in pp collisions at $13 \mathrm{TeV}$, http://cds.cern.ch/ record $/ 2205270$. 
[66] W.-Y. Keung and G. Senjanovic, Phys. Rev. Lett. 50, 1427 (1983).

[67] A. Datta, M. Guchait, and A. Pilaftsis, Phys. Rev. D 50, 3195 (1994).

[68] F. M. L. Almeida, Jr., Y. do Amaral Coutinho, J. A. Martins Simoes, and M. A. B. do Vale, Phys. Rev. D 62, 075004 (2000).

[69] O. Panella, M. Cannoni, C. Carimalo, and Y. N. Srivastava, Phys. Rev. D 65, 035005 (2002).

[70] T. Han and B. Zhang, Phys. Rev. Lett. 97, 171804 (2006).

[71] F. del Aguila, J. A. Aguilar-Saavedra, and R. Pittau, J. High Energy Phys. 10 (2007) 047.

[72] K. Huitu, S. Khalil, H. Okada, and S. K. Rai, Phys. Rev. Lett. 101, 181802 (2008).

[73] A. Atre, T. Han, S. Pascoli, and B. Zhang, J. High Energy Phys. 05 (2009) 030.

[74] A. Das, P. Konar, and S. Majhi, J. High Energy Phys. 06 (2016) 019.

[75] R. Ruiz, M. Spannowsky, and P. Waite, Phys. Rev. D 96, 055042 (2017).

[76] A. Abulencia et al. (CDF Collaboration), Phys. Rev. Lett. 98, 221803 (2007).

[77] S. Chatrchyan et al. (CMS Collaboration), Phys. Lett. B 717, 109 (2012).

[78] Tech. Rep. ATLAS-CONF-2012-139, CERN, Geneva, 2012.

[79] V. Khachatryan et al. (CMS Collaboration), Phys. Lett. B 748, 144 (2015).

[80] G. Aad et al. (ATLAS Collaboration), J. High Energy Phys. 07 (2015) 162.

[81] V. Khachatryan et al. (CMS Collaboration), J. High Energy Phys. 04 (2016) 169.

[82] F. del Aguila, J. de Blas, and M. Perez-Victoria, Phys. Rev. D 78, 013010 (2008).

[83] E. Akhmedov, A. Kartavtsev, M. Lindner, L. Michaels, and J. Smirnov, J. High Energy Phys. 05 (2013) 081.
[84] L. Basso, O. Fischer, and J. J. van der Bij, Europhys. Lett. 105, 11001 (2014).

[85] S. Antusch and O. Fischer, J. High Energy Phys. 10 (2014) 094.

[86] M. Aaboud et al. (ATLAS Collaboration), New J. Phys. 18, 093016 (2016).

[87] A. M. Sirunyan et al. (CMS Collaboration), J. High Energy Phys. 07 (2017) 121.

[88] G. Aad et al. (ATLAS Collaboration), J. High Energy Phys. 06 (2014) 035.

[89] M. Aaboud et al. (ATLAS Collaboration), J. High Energy Phys. 09 (2017) 084.

[90] G. Aad et al. (ATLAS Collaboration), Eur. Phys. J. C 76, 259 (2016).

[91] T. Sjostrand, S. Mrenna, and P. Z. Skands, J. High Energy Phys. 05 (2006) 026.

[92] J. de Favereau, C. Delaere, P. Demin, A. Giammanco, V. Lemaître, A. Mertens, and M. Selvaggi (DELPHES 3 Collaboration), J. High Energy Phys. 02 (2014) 057.

[93] M. Selvaggi, J. Phys. Conf. Ser. 523, 012033 (2014).

[94] A. Mertens, J. Phys. Conf. Ser. 608, 012045 (2015).

[95] M. Cacciari, G. P. Salam, and G. Soyez, J. High Energy Phys. 04 (2008) 063.

[96] M. Cacciari and G. P. Salam, Phys. Lett. B 641, 57 (2006).

[97] M. Cacciari, G. P. Salam, and G. Soyez, Eur. Phys. J. C 72, 1896 (2012).

[98] Tech. Rep. ATL-PHYS-PUB-2015-022, CERN, Geneva, 2015.

[99] M. Drees, H. Dreiner, D. Schmeier, J. Tattersall, and J. S. Kim, Comput. Phys. Commun. 187, 227 (2015).

[100] D. Dercks, N. Desai, J. S. Kim, K. Rolbiecki, J. Tattersall, and T. Weber, Comput. Phys. Commun. 221, 383 (2017).

[101] A. L. Read, J. Phys. G28, 2693 (2002). 